\title{
基于 GIS 数据的快速地图制图技术研究
}

\section{Research on the Technology of Fast Map Drawing Based on GIS Data \\ 邵奎明 郭向京}

Kuiming Shao Xiangjing Guo

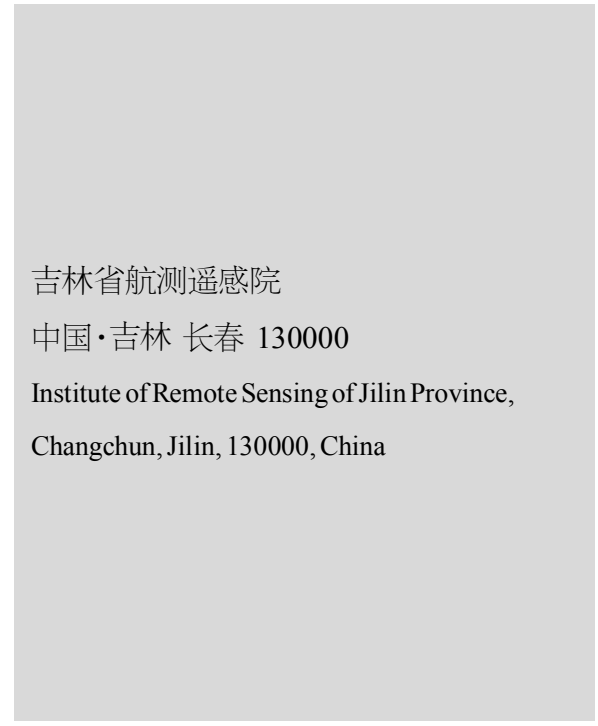

【摘要】在现代化社会的发展中, 地图制图行业发展十分迅速, 社会各界越来越关注地理 信息工程的发展。相关部门需要引进更多先进技术, 利用 GIS 数据确保数据加工流程的实 用性、科学性, 为地图制图工作的顺利实施提供支持。论文主要对基于 GIS 数据的快速地 图制图技术进行了研究。

【Abstract \In the development of modern society, cartography industry is developing rapidly, and more and more people are paying attention to the development of geographic information engineering. Relevant departments need to introduce more advanced technologies, use GIS data to ensure the practicability and scientificity of data processing process, and provide support for the smooth implementation of cartography. This paper mainly studies the technology of rapid cartography based on GIS data.

【关键词】GIS; 空间数据; 地图制图

【Keywords \GIS; spatial data; cartography

【DOI】10.36012/se.v1i2.921

\section{1 引言}

地图作为一种信息载体, 其能够对生存环境进行研究和 描述, 不仅是简单地表达地类要素, 还融合了科学技术, 是一 种必需的应用工具。在科学技术水平快速提升的大背景下, 科 学信息数据越来越多,在应用和制作地图过程中, 由于地图到 空间数据表达之间存在很多问题，导致地图信息缺失问题严 重 ${ }^{[1]}$ 。在地图空间数据到地图表达全过程中, 需要引进更多科 学技术完善各项信息,为地图信息的完整性提供支持。

\section{GIS 空间数据的相关内容}

GIS 空间数据是为地理研究和社会服务建立的计算机系 统, 能够全面处理和分析空间数据。GIS 技术的应用基础是空 间数据库, 在实际运行过程中, 可根据计算机技术采集、管理、 操作、分析和模拟各项空间数据, 以获取地理信息的空间动态 效果。在地理制图发展中, GIS 空间数据会得到更加广泛的应 用,其具有空间、属相、时间特征,表现了空间的实质现象。

\section{3 基于 GIS 数据的快速地图制图技术}

\section{1 明确数据标准}

为了更好地将建库和制图进行有效融合, 相关部门需要 明确数据生成、建库和制图相关标准。在数据生成前期,相关 人员需要获取更多 GIS 数据以外的相关制图信息, 确保后期 地图绘制工作的有效性。另外, 还需要引进综合数据模型, 其 主要涉及空间数据、属性数据和制图数据, 并根据实际情况确 定相关标准, 合理地填充各项数据。

\section{2 关键技术分析}

\subsection{1 数据篮选}

编图人员需要做好制图实体标注工作, 根据实际出图要 求, 引进 GIS 技术对各项数据进行筛选。例如, 在交通网络图 制作过程中, 编图人员需要将地理背景信息和道路信息进行 融合, 如行政区域划分、地名、水系、山峰、道路等, 保证原有数 据和各项要素规范的一致性, 并确保后续符号化处理工作的 统一性。 
地图学 Cartography

\subsection{2 地图投影}

在跨越度范围大的地图制图过程中，为了满足长度和面 积等各类变形要求,编图人员需要掌握实际情况, 以此为基础 合理地选择特定的地图投影，一般正轴双标准纬线为基础的 众多角圆雉投影方式, 满足了地图制图过程中的面积、长度等 变形需求, 为地图制度的有效性提供了支持。

\section{2 .3 数据格式转变}

数据格式转变指的是 GIS 数据到 MAPGIS 的转变, 常见 的两种形式是直接数据交换、访问中间数据 ${ }^{[2]}$ 。其中, 直接数 据交换指的是利用编写程序直接读取 GIS 数据，并根据自定 义数据格式输出, 需要利用变化坐标、层定义、数据读写方式 实现这一目标，访问中间数据是直接打开 GIS 数据的中间数 据格式。

\section{3 智能化标注}

在地图制图过程中, GIS 空间数据和地图制图的融合, 能 够实现智能化标注。地图制图智能化标注能力的主要表现是 能够有效地解决制图中点、线、面自动标注的标注位置、冲突 处理等问题。在地图制图过程中, GIS 空间数据和制图技术的 融合有利于控制标注位置和间隔字符，严格控制和处理标注 放置策略、标注冲突, 实现图面整饰的效果, 满足自由缩放的 各项需求, 提高地图应用的准确性、有效性。首先, 准确地控制 标注位置、偏移量、间隔标注等内容, 无需在前期完成数据预 处理工作; 其次, 解决标注放置策略和标注冲突问题,需要满 足标注大小自由缩放的需求; 最后, 标注转变成注记后, 需要 针对注记进行拆分、移动、缩放、删除和修改等各项编辑。

\section{4 丰富制图表达}

为了丰富制图表达, 编图人员需要做好以下工作: 第一, 根据地理特征分类和组织空间数据，重点要合理地控制符号 和符号之间的关系,确保地理要素线划的美观性,还需要合理 地控制图层叠加顺序; 第二, 在获取相应的数据符号后, 基础 地形图需要显现相同土层中各个地物类对象的上下关系; 第
三,针对点、线等有向地物制图表达的过程中,需要根据角度 方向挂接符号; 第四, 在遇到小路、河流相交的情况时, 相关技 术人员需要利用虚线符号实现相交的制图规则; 第五, 虚线符 号怪点位置必须是实部; 第六, 在桥梁和道路位置, 需要满足 桥梁符号和道路方向一致的原则; 第七, 在运用等高线、河流 等线状符号时,需要确保符号线条的光滑性。

\section{5 设定制图比例尺}

为了更好地转变各项数据, 相关人员需要在 Geoway 软件 下设定比例尺和地图投影[3]。例如, 1:2000 缩编成 1:5000,1:5000 缩编成 1:10000,1:10000 缩编成 1:50000, 以此为基础缩编空间 数据, 并对各项空间数据进行符号化处理, 实现 1:50000 的制 度数据效果。

\section{4 结语}

综上所述, 在新时期的快速发展中, 很多先进的科学技术 在各个行业的发展中得到了有效应用, GIS 地理信息系统具 备完善的理论体系,现已成为高效的空间信息系统, GIS 空间 数据和地图制图的有效融合逐渐成为地图制图中的关键技 术, 有效地提升了人民群众对地图的理解程度, 使相关研究人 员更加关注地图制图技术，尤其是 GIS 空间数据在地图制图 技术中的应用，提升了地图制图的高效性，实现了地图制图技 术的信息化,为制图行业的发展提供了保障。

\section{参考文献}

[1]胡国红.基于 GIS 的地图制图技术研究[J].低碳世界,2019,9 (7):354-355

[2]邱源.从 GIS 到平面地图制图技术路线浅谈一基于 Adobe Illustrator+Avenza MAPublisher 的空间数据库制图技术研究 [J]. 经纬 天地,2016(6):68-71.

[3]潘凤桃, 廖勇.GIS 数据与制图数据一体化建库技术的实现 $[\mathrm{J}]$. 建筑工程技术与设计,2017(8):57. 